\title{
Robust PID Control Design via Mixed Particle Swarm Optimization Algorithm and Gap Metric
}

\author{
Xinghong Qiao ${ }^{1,2}$, Fei Luo ${ }^{1}$, Yuge $\mathrm{Xu}^{1}$ \\ ${ }^{1}$ College of Automation Science and Engineering, South China University of \\ Technology, Guangzhou 510641, China \\ ${ }^{2}$ Guangzhou Institute of Energy Testing, Guangzhou 511447, China \\ qiaoxinghong@126.com
}

\begin{abstract}
This paper proposes a novel tuning approach for robust proportional-integralderivative (PID) controller based on $H_{\infty}$ loop shaping synthesis in combination with gap metric and Particle Swarm Optimization (PSO) algorithm. Different from the traditional research, the controller is designed through the search region constrained by $H_{\infty}$ loop shaping synthesis and gap metric theorem. PSO algorithm is used for tuning the robust PID controller parameters based on the underlying constrained optimization problems without resolving complex arithmetical calculations. The control technique is applied for the robust controller design so as to get a low order structured controller and achieve robust performance and the ability of restraining disturbance. The simulation shows that the proposed method can character the set of all values of the controller parameters that guarantees the robust stability with any supposed accuracy and achieves favorable control performance for uncertain systems.
\end{abstract}

Keywords: gap metric, robust controller, PSO algorithm, model uncertainty, loop shaping

\section{Introduction}

A feedback control system consists of two basic parts: the controlled object plant and controller. In the analysis and synthesis of the feedback uncertain systems, many control techniques have been developed. The classical approach of the robust controllers for nonlinear uncertain systems designed by using the $\mathrm{H}_{2}, \mathrm{H}_{\infty}$, or $\mu$ formulations can produce extremely fragile controllers [1]. The robust controller is designed for structured uncertain systems by singular value synthesis [2], but this kind of control design is computationally intractable [3]. The $\mathrm{H}_{\infty}$ loop shaping design procedure (LSDP) is an effective method to design robust controller [4] and is being used in a wide range of applications in industry. The gap metric was introduced into control field to study of robustness properties of uncertainty systems by Zames and El-Sakkary [5, 6]. The graph metric and similar metric for normalized coprime-factorized models was introduced by Vidyasagar [7]. The v-gap metric was proposed by Vinnicombe [8]. Georgiou and Smith proved that the problem of robustness optimization in the gap metric was equivalent to robustness optimization for perturbations in normalized coprime factors [9]. Qiu and Davison proposed pointwise gap metrics for uncertain systems to study stability robustness [10]. Although these metrics are different numerical, they are the same topology. The gap metric has been widely used to design the robust controller in the presence of uncertainty plant [11-14].

However, the resulting controllers designed by the above approaches are computationally intractable and the orders of $\mathrm{H}_{\infty}$ loop shaping controllers are high. The stochastic optimization for tuning of PID controller method is a worthwhile direction to 
overcome the difficulties. The paper is concerned with the development of robust PIDcontrollers based on $\mathrm{H}_{\infty}$ loop shaping synthesis in combination with gap metric. In our approach, the $\mathrm{H}_{\infty}$ loop shaping and v-gap metric which is maximal gap metric between the nominal system model and any other models are used to define a family of robustly stable controllers. However, the problem arises the computation of optimal solutions. This constrained optimization problems can be solved by using Particle Swarm Optimization (PSO). PSO algorithm has demonstrated the ability to deal with non-convex optimization problems $[16,17]$.

The main goal of this paper is to further develop robust control synthesis approaches for uncertain systems. The controller designed based on conventional mathematical and analytical methods is usually computationally intractable. In order to simplify mathematical calculations, the v-gap theorem and $\mathrm{H}_{\infty}$ specifications in combination with PSO algorithm are used to solve the underlying constrained optimization problems. The rest of this paper is structured as follows. In Section 2, the proposed PID controller in state-space structure and the conventional $\mathrm{H}_{\infty}$ loop shaping are illustrated. The gap measurement and robust stability analysis are constructed in Section 3. In Section 4, PSO algorithm is briefly introduced and used to solve the optimization problem constrained by the v-gap theorem and $\mathrm{H}_{\infty}$ specifications. The simulation study is carried out by Matlab/Simulink to verify the comparatively strong robust and the ability of restraining disturbance in Section 5. Finally, the concluding remarks are drawn in Section 6.

\section{Problem Setting and Derivation of Constraint}

\subsection{PID Controller Structure}

The proportional-integral-derivative (PID) controller is defined as follows [18]:

$$
K_{P I D}(s)=K_{P}+K_{I} \frac{1}{S}+K_{D} \frac{s}{1+\tau s}=\left[\begin{array}{cc:c}
0 & 0 & K_{I} \\
0 & -\frac{1}{\tau} I & -\frac{1}{\tau^{2}} K_{D} \\
\hdashline I & I & K_{P}+\frac{1}{\tau} K_{D}
\end{array}\right]=\left[\begin{array}{c:c}
A_{K} & B_{K} \\
\hdashline C_{K} & D_{K}
\end{array}\right]
$$

where $K_{P}, K_{I}, K_{D}$ are the proportional, integral and derivative matrix gains, respectively, and $\tau$ is the time constant. PID controller can be used a simple firstorder low-pass filter to ensure the properness and physical realizability of PID controller. The matrices $A_{K}, B_{K}$, and $C_{K}$ must satisfy the structures described in (1); the matrix $D_{K}$ must satisfy the well-posedness condition.

\section{2. $\mathrm{H}_{\text {inf }}$ Loop Shaping}

The robust control design method based a combination of loop shaping and robust stabilization was proposed in McFarlane and Glover [19, 20]. In this framework, the open-loop scaled plant $\mathrm{G}(\mathrm{s})$ is shaped with the pre-compensator $W_{1}(s)$ and postcompensator $W_{2}(s)$ as shown in Figure 1 . The $W_{1}(s)$ and $W_{2}(s)$ are chosen so that the weighted plant is sufficiently at all frequencies, typically a large gain at low frequencies for good robust stability and a small gain at high frequencies for disturbance attenuation. 


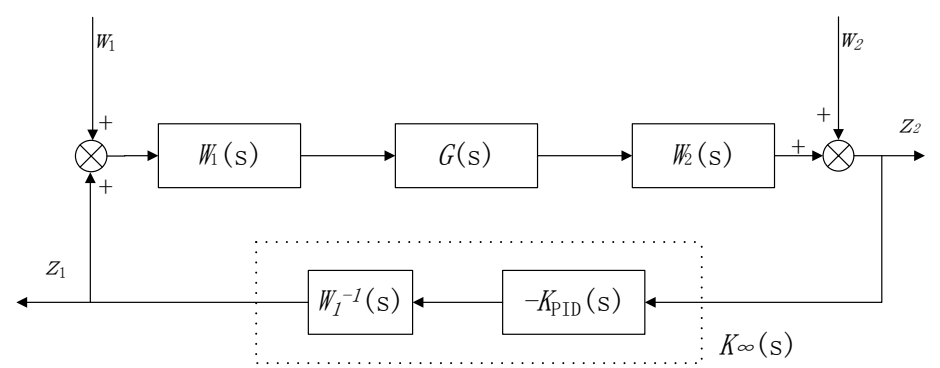

Figure 1. Standard Block Diagram of $\mathrm{H}_{\mathrm{inf}}$ Loop Shaping Control Method

The transfer functions from disturbances $w_{1}$ and $w_{2}$ to the outputs $z_{1}$ and $z_{2}$ can be written as follows:

$$
\left[\begin{array}{l}
z_{1} \\
z_{2}
\end{array}\right]=T_{z w}\left(K_{\infty}\right)\left[\begin{array}{l}
w_{1} \\
w_{2}
\end{array}\right]
$$

where $T_{z w}=\left[\begin{array}{c}I \\ -W_{1}^{-1} K_{P I D}\end{array}\right]\left(I+W_{2} G K_{P I D}\right)\left[\begin{array}{ll}W_{2} G W_{1} & I\end{array}\right]$. The controlled system with the proposed PID controller should achieve perfect dynamic performance and strong robustness. The design problem can be expressed for solving a minimization problem as follows:

$$
\min _{\text {stab } K_{P I D}}\left\|T_{z w}\right\|_{\infty}=\min _{\text {stab } K_{P I D}}\left\|\left[\begin{array}{c}
I \\
-W_{1}^{-1} K_{P I D}
\end{array}\right]\left(I+W_{2} G K_{P I D}\right)\left[\begin{array}{ll}
W_{2} G W_{1} & I
\end{array}\right]\right\|_{\infty}
$$

The above numerical constrained optimization is equivalent to maximise stability margins for stabilising PID controllers. The non-convex minimization problems can not be solved easily. However, a solution can be search based on evolutionary algorithm if the optimisation problem is structured in the state-space matrix inequalities. The statespace realization for the transfer matrix from $w_{1}$ and $w_{2}$ to $z_{1}$ and $z_{2}$ can be obtained as [21].

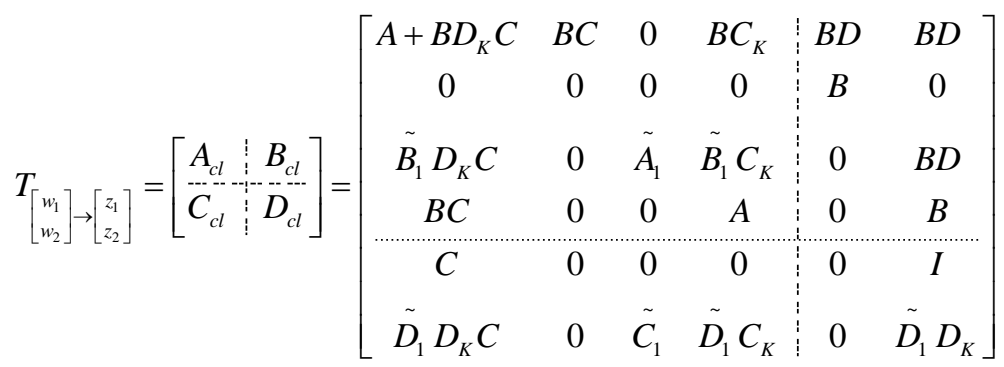

where $W_{1}=\left[\begin{array}{c:c}A_{1} & B_{1} \\ \hdashline C_{1} & D_{1}\end{array}\right], W_{1}^{-1}=\left[\begin{array}{c:c}\tilde{A}_{1} & \tilde{B}_{1} \\ \hdashline \tilde{C} & \tilde{D}_{1}\end{array}\right], W_{2} G=\left[\begin{array}{c:c}A & B \\ \hdashline C & D\end{array}\right]$.

\section{Gap Measurement and Robust Stability Analysis}

\subsection{Gap Measurement and Geometry Meanings}

The controller for uncertain systems should be satisfied not only adequate level of performances but also robust metrics. The $v$-gap can offer a sensible measure of uncertain system. The spherical distance between $c_{1}$ and $c_{2}$, denoted by $\theta\left(c_{1}, c_{2}\right)$, is defined as the shortest length of an arc on Riemann sphere connecting $\varphi\left(c_{1}\right)$ and $\varphi\left(c_{2}\right)$, and the center of the Riemann sphere as shown in Figure 2. 


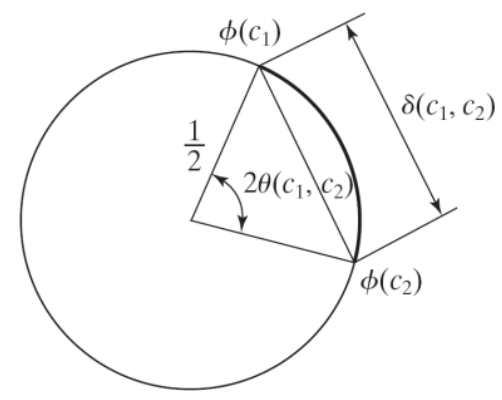

Figure 2. The Relationship Between $\delta\left(c_{1}, c_{2}\right)$ and $\theta\left(c_{1}, c_{2}\right)$

$$
\begin{gathered}
\delta\left(c_{1}, c_{2}\right)=\left\|\varphi\left(c_{1}\right)-\varphi\left(c_{2}\right)\right\|=\frac{\left|c_{1}-c_{2}\right|}{\sqrt{1+c_{1}^{2}} \sqrt{1+c_{2}^{2}}} \\
\theta\left(c_{1}, c_{2}\right)=\arcsin \delta\left(c_{1}, c_{2}\right)=\arcsin \frac{\left|c_{1}-c_{2}\right|}{\sqrt{1+c_{1}^{2}} \sqrt{1+c_{2}^{2}}}
\end{gathered}
$$

The transfer functions $G_{i}(s), i=1,2$, of any single-input-single-output (SISO) systems can be given as the following:

$$
G_{i}(s)=\frac{b_{i}(s)}{a_{i}(s)}
$$

where $a_{i}(s), b_{i}(s), i=1,2$, are polynomials. The chordal distance between two systems $G_{1}(s)$ and $G_{2}(s)$ is presented as

$$
\delta\left(G_{1}, G_{2}\right)=\frac{\left|a_{2} b_{1}-a_{1} b_{2}\right|}{\sqrt{\left|a_{1}\right|^{2}+\left|b_{1}\right|^{2}} \sqrt{\left|a_{2}\right|^{2}+\left|b_{2}\right|^{2}}}
$$

The normalized right coprime factorizations of multiple-input-multiple-output (MIMO) systems $G_{1}(s)$ and $G_{2}(s)$ are $\left(D_{1}, N_{1}\right)$ and $\left(D_{2}, N_{2}\right)$, respectively. Then the gap between $G_{1}(s)$ and $G_{2}(s)$ can be calculated by the following formula [23]:

$$
\delta\left(G_{1}, G_{2}\right)=\max \left\{\delta\left(G_{1}, G_{2}\right), \delta\left(G_{2}, G_{1}\right)\right\}
$$

where $\delta\left(G_{1}, G_{2}\right)=\inf _{Q_{1} \in M\left(H_{\infty}\right)}\left\|\left[\begin{array}{c}D_{1} \\ N_{1}\end{array}\right]-\left[\begin{array}{c}D_{2} \\ N_{2}\end{array}\right] Q_{1}\right\|, \delta\left(G_{2}, G_{1}\right)=\inf _{Q_{1} \in M\left(H_{\infty}\right)}\left\|\left[\begin{array}{c}D_{2} \\ N_{2}\end{array}\right]-\left[\begin{array}{c}D_{1} \\ N_{1}\end{array}\right] Q_{2}\right\|$, $M\left(H_{\infty}\right)$ denotes the set of all matrices with elements in $H_{\infty}$.

All models denoted by $\mathcal{B}_{\delta}(G, r)$ with the graph uncertainty centered at nominal plant $G$ with radius $r$ are defined by

$$
\mathcal{B}_{\delta}(G, r)=\left\{G_{1}: \delta\left(G_{1}, G\right) \leq r\right\}
$$

where $\delta(.,$.$) is the spherical distance, \mathrm{G}$ is nominal system, $\mathrm{r}$ is uncertain radius. Similarly, $\theta(.,$.$) is the spherical distance. A set of uncertainty models \mathcal{B}_{\theta}(G, r)$ is defined by

$$
\mathcal{B}_{\theta}(G, r)=\left\{G_{1}: \theta\left(G_{1}, G\right) \leq \arcsin r\right\}
$$

\subsection{Robust Stability Based on Gap Measurement}

Consider the standard feedback configuration depicted in Figure 3. The standard feedback is stable if and only if the following equation is correct.

$$
T_{e w}=\left[\begin{array}{cc}
(1-C P)^{-1} & C(1-P C)^{-1} \\
P(1-C P)^{-1} & (1-P C)^{-1}
\end{array}\right] \in R H_{\infty}
$$


where $T_{w e}$ is the transfer function from $w=\left[\begin{array}{l}w_{1} \\ w_{2}\end{array}\right]$ to $z=\left[\begin{array}{l}z_{1} \\ z_{2}\end{array}\right]$.

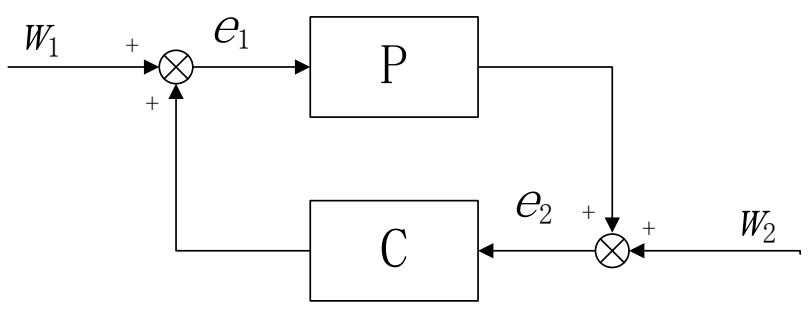

Figure 3. Standard Feedback Configuration

If the feedback system is stable for load disturbances, it is necessary to minimize the effects of $T_{w e}$, which means that the cost minimization function in (13) is solved.

$$
\rho=\left\|\left[\begin{array}{l}
I \\
P
\end{array}\right](I-C P)^{-1}\left[\begin{array}{ll}
I & -C
\end{array}\right]\right\|_{\infty}
$$

The objective is to find a stabilizing controller ensuring the best possible disturbance

rejection. The corresponding optimal $\mathrm{H}_{\infty}$ controller is the solution for the following optimization problem:

$$
\text { minimize } \gamma
$$

subject to $\left\|T_{e w}\right\|_{\infty} \leq \gamma$

Alternately, it can be defined as

$$
b_{P, C} \stackrel{\Delta}{=} \frac{1}{\rho}=\left\|\left[\begin{array}{l}
I \\
P
\end{array}\right](I-C P)^{-1}\left[\begin{array}{ll}
I & -C
\end{array}\right]\right\|_{\infty}^{-1}
$$

$b_{P, C}$ is called the robust stability radius, which was introduced by Tryphon T. Georgiou and Malcolm C. Smith in terms of the controller and the plant [9]. It is regarded as the generalized stability margin.

Lemma1[21] The uncertain gap measure $\delta\left(P_{1}, P_{2}\right)$ and the stability margin $b_{P, C}$ can be related by the following inequality:

$$
\arcsin \left(b_{P_{2}, C}\right) \geq \arcsin \left(b_{P_{1}, C}\right)-\arcsin \delta\left(P_{1}, P_{2}\right)
$$

Theorem1 The controller $\mathrm{C}$ stabilizes any given system $\tilde{P}$ if and only if $\delta(P, \tilde{P})<1 / \gamma_{\min }$, where $\gamma_{\min }$ is the minimal $\mathrm{H}_{\infty}$ norm of the closed-loop transfer matrix $T_{e w}, P$ is nominal system.

Proof: According to the Lemma1, the following inequality can be get:

$$
b_{P_{2}, C} \geq b_{P_{1}, C}-\delta\left(P_{1}, P_{2}\right)
$$

Since the closed-loop system $\left[P_{2}, C\right]$ is stable if $b_{P_{2}, C}>0$, the inequality $b_{P_{1}, C}>\delta\left(P_{1}, P_{2}\right)$ exists. On the other, the controller $\mathrm{C}$ can stabilize $P_{1}$ and also stabilize $P_{2}$. The $P_{1}$ and $P_{2}$ are any given systems.

\section{Control Designer Based on PSO Algorithm}

\subsection{Introduction to PSO [24]}

Particle swarm optimization(PSO) algorithm, which stems from the simulation of birds flock's looking for food, is a swarm intelligence-based evolutionary computing. Consider the following optimization problem: 


$$
\operatorname{Min}_{x \in \chi} f(x)
$$

The typical PSO algorithm includes the following steps, which is repeated either a certain number of times or until a particular stopping condition.

1. Initialization. PSO is initialized with a population of random particles by assigning random position and velocities inside the problem space. It combines local search and global search activities, possessing high search efficiency.

2. Swarm evolution. Each particle has fitness values which is evaluated by the fitness function optimized, and has velocity which direct the flying of the particles.

3. After finding the two best values, the particle updates best fitness and positions with following equation

$$
\begin{aligned}
& \hat{x}_{\text {best }, i}(t+1)=\hat{x}_{\text {best }, i}(t), \text { if } f\left[x_{i}(t+1)\right]>\hat{x}_{\text {best }, i}(t) \\
& \hat{x}_{\text {best }, i}(t+1)=x_{i}(t+1) \text {,if } f\left[x_{i}(t+1)\right] \leq \hat{x}_{\text {best }, i}(t) \\
& g_{\text {best }}(t)=\min \left\{\hat{x}_{\text {best }, i}(t)\right\}
\end{aligned}
$$

where $i \in[1,2, \cdots, n]$ and $n>1$.

4. The velocities and positions of all particles are updated. Fitness evaluation is performed by calculating objective function. The best fitness and positions are updated, which are responsible for the optimization ability of PSO algorithm. The positions and velocities of all the particles are updated through the following equations:

$$
\begin{aligned}
& v_{i}(t+1)=\omega v_{i}(t)+c_{1} r_{1}\left[\hat{x}_{i}(t)-x_{i}(t)\right]+c_{2} r_{2}\left[g(t)-x_{i}(t)\right] \\
& x_{i}(t+1)=x_{i}(t)+v_{i}(t+1)
\end{aligned}
$$

where $i, v_{i}(t)$ and $x_{i}$ represent the index, velocity and position of the particle, respectively.

\subsection{Largest $v$-gap Metric Based on PSO Algorithm}

PID controller based on PSO algorithm structure diagram is shown in Figure 4. The controller is mainly for the following three parts to carry on the design.

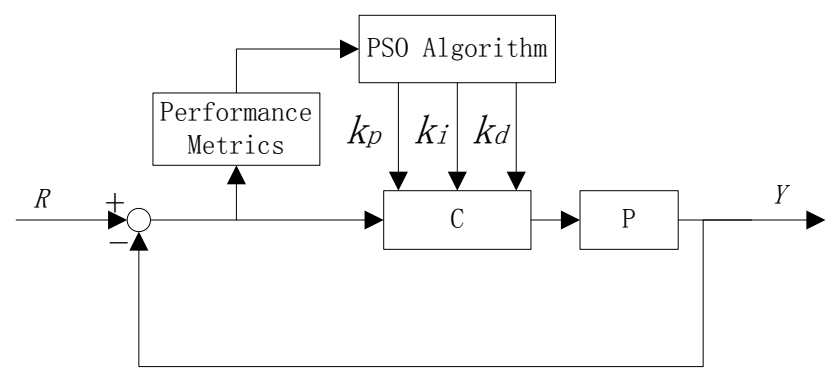

Figure 4. Structure Diagram of PID Control System Based on PSO

(1) The largest gap metric $\delta_{w c}$ between the nominal system and any given system $\tilde{P}$ is calculated by mixing particle swarm optimization algorithm and Matlab command "gapmetric".

(2) According to the experience of the project, the designers explore and design the search space as $D=\left\{x \in R^{3}: x_{i} \leq x_{i} \leq \bar{x}_{i} \quad i=1,2,3\right\}$. 
(3) Fixed-order $\mathrm{H}_{\infty}$ synthesis with PSO the problem can be solved as follows. At iteration $k$, after moving the swarm according to (20-24), do for each particle:

a. Build PID controller by $\left[\begin{array}{c:c}A_{K} & B_{K} \\ \hdashline C_{K} & D_{K}\end{array}\right]$,

b. Build $\left[\begin{array}{c:c}A_{c l} & B_{c l} \\ \hdashline C_{c l} & D_{c l}\end{array}\right]$ according to (1)

c. Evaluate $\lambda_{A c l}=\operatorname{Re}\left(\lambda_{i}\left(A_{c k}\right)\right)$

If $\lambda_{\text {Acl }} \geq 0, f\left(x_{p}^{k}\right)=\inf$,

else $f\left(x_{p}^{k}\right)=\gamma$

d. Solve $\gamma_{\min }$ by PSO algorithm

e. if $\delta_{w c} \geq 1 / \gamma_{\min }$ the controller $\mathrm{C}$ don't stabilize any given system $\tilde{P}$ else the controller stabilize any given system $\tilde{P}$

\section{A Numerical Example}

Consider the uncertain system shown as Figure 5 [25].

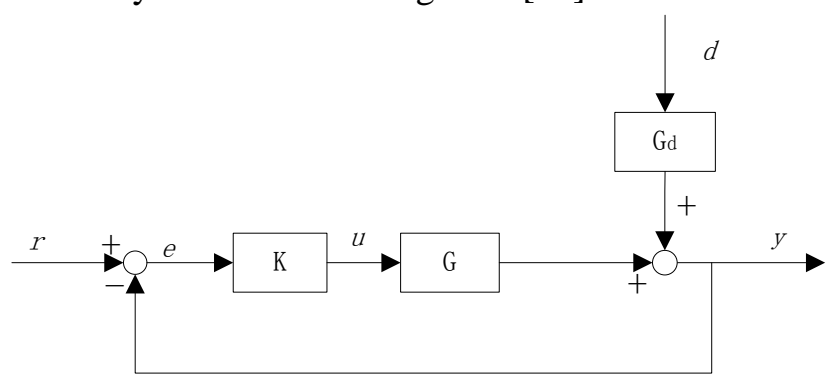

Figure 5. Block Diagram of a Feedback Control System

$$
G(s)=\frac{k}{(a s+1)(b s+1)^{2}} \quad G_{d}(s)=\frac{100}{10 s+1}
$$

Assume that the coefficients $\mathrm{k}$, $\mathrm{a}$ and $\mathrm{b}$ of the denominator lie within the following bounds, where $180 \leq k \leq 220,9 \leq a \leq 11,0.045 \leq b \leq 0.055$. The loop shaping weighting functions $W_{1}$ and $W_{2}$ for the nominal systems are chosen as follows:

$$
W_{1}=\frac{0.75 s+4.001}{s+0.001} \quad W_{2}=1
$$

PID controller is given as follows:

$$
K(s)=x_{1}+\frac{x_{2}}{s}+\frac{x_{3} s}{0.01 s+1}
$$

where $x:=\left(x_{1}, x_{2}, x_{3}\right)=\left(k_{p}, k_{i}, k_{d}\right)$ and the search space is set as $\mathrm{D}:=\left\{\mathrm{x} \in \mathrm{R}^{3}:(-3,-3,-3,)^{\mathrm{T}}\right.$ $\left.<x<(8,6,5)^{\mathrm{T}}\right\}$. The above algorithm is applied for the system (23) to find the parameters: $k_{p}=0.6, k_{i}=1.3682, k_{d}=0.016, b_{P, C}=3.097 \gamma_{\min }=0.295$.The controller is obtained as following:

$$
K_{1}(s)=0.6+\frac{1.3682}{s}+\frac{0.016 s}{0.01 s+1}
$$

The corresponding robustness margin

is $b_{P, C}=1 / 3.097=0.323>\delta_{v}(P, \tilde{P})=0.295$. Therefore the controller is guaranteed to be stable 
against parametric uncertainties. The step responses of nominal system and various parameters system are shown in Figure 6 and Figure 7, respectively. As shown in Figure 7 shows, any given system can be controlled sufficiently well with the synthesis method.

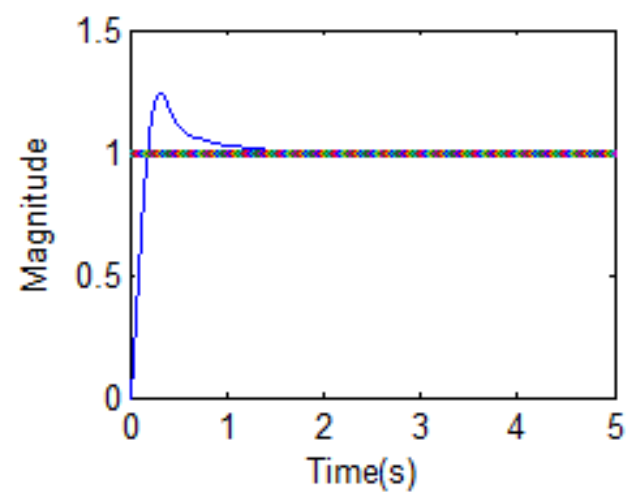

Figure 6. Step Response of Nominal System

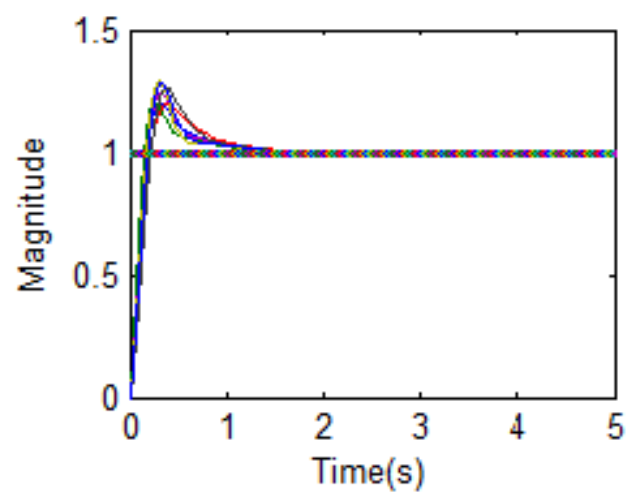

Figure 7. Step Response of Uncertain System

The full order controller is designed by the standard $\mathrm{H}_{\infty}$ loop shaping design method $[25,26]$ as following:

$$
K_{2}(s)=\frac{123.8 s^{4}+6080 s^{3}+9.644 \times 10^{4} s^{2}+5.212 \times 10^{5} s+8.584 \times 10^{5}}{s^{5}+127.8 s^{4}+5853 s^{3}+1.344 \times 10^{5} s^{2}+5.668 \times 10^{5} s+566.7}
$$

The unit step and loop shaping in the disturbance response for the uncontrolled case, full order controller and synthesis PID algorithm are shown in Figure 8 and Figure 9, respectively. The solid line, the dash-dot line and dash line present the uncontrolled case, full order controller and synthesis PID algorithm. Table 1 shows the results in detail. The gain margin $(\mathrm{GM})$ is improved from 3.481 (for the full order case) to 19.700 (for synthesis PID algorithm), and the phase margin (PM) is reduced slightly from $51.492^{\circ}$ to $50.920^{\circ}$. The gain crossover frequency is reduced 10.257 to $9.271(\mathrm{rad} / \mathrm{s})$. The results show that the synthesis PID algorithm and full order controller are robust to the uncertain-but-bounded system and the influent disturbances. But the synthesis PID algorithm controller is lower order and computationally tractable. Furthermore according to Table 1, the time-domain synthesis PID algorithm is quite competitive in comparison with the full order case.

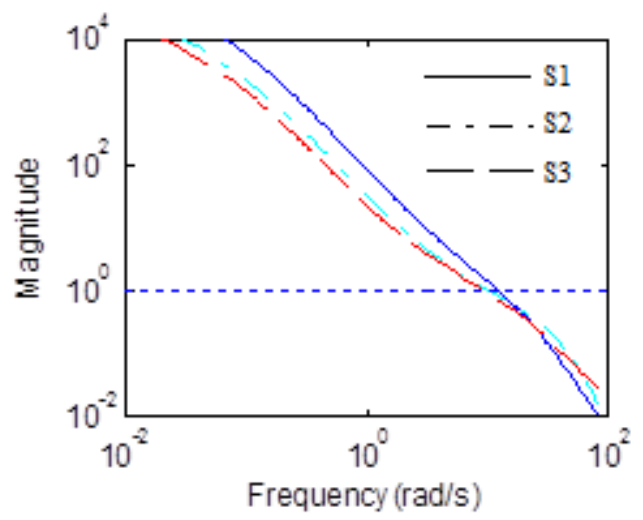

Figure 8. Loop Shapes

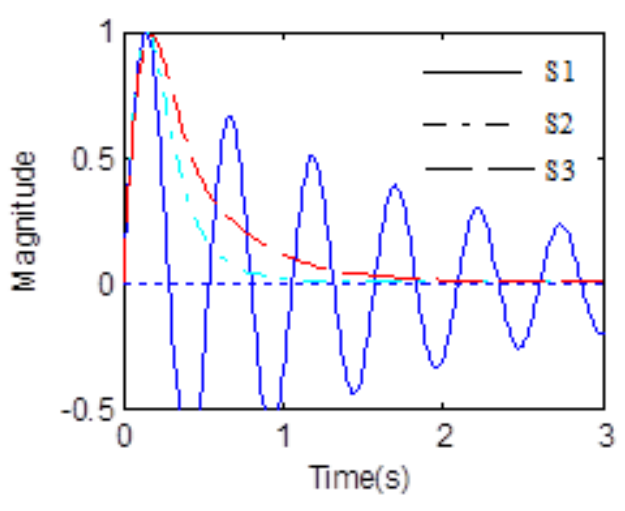

Figure 9. The Unit Step in the Disturbance Response 
Table 1. Comparison of the Performance of Different Controllers

\begin{tabular}{|c|c|c|c|c|}
\hline $\begin{array}{l}\text { performance } \\
\text { method }\end{array}$ & $\gamma_{\text {min }}$ & gain margin & phase margin & crossover frequency \\
\hline the uncontrolled case & --- & 1.618 & $13.179^{O}$ & $13.733 \mathrm{rad} / \mathrm{s}$ \\
\hline the full order case & 2.593 & 3.481 & $51.492^{\circ}$ & $10.257 \mathrm{rad} / \mathrm{s}$ \\
\hline synthesis PID algorithm & 3.097 & 19.700 & $50.920^{\circ}$ & $9.271 \mathrm{rad} / \mathrm{s}$ \\
\hline
\end{tabular}

\section{Conclusions}

In this paper, a synthesizing PID robust controller based on $\mathrm{H}_{\infty}$ loop shaping in combination with $v$-gap metric and PSO algorithm is developed. Generally, the robust controller design problem using conventional mathematical and analytical methods is known to be computationally intractable and the order of the controller is high. PSO algorithm is used for tuning the robust PID controller parameters without resolving complex arithmetical calculations by minimizing a fitness function. The control algorithm is applied for the robust controller design so as to achieve robust performance. Simulate and experiment show that the control technology has comparatively strong robust and the ability of restraining disturbance. For instance, the proposed controller is more stable to the uncontrolled case and the order of the controller is lower to the full order case. PSO algorithm is quite generic and can be replaced by any other evolutionary algorithm. The control technique may provide a novel method for the robust PID design.

\section{Acknowledgements}

This work is supported by The Natural Science Foundation of Guangdong Province (10151064101000075); the project on the Science \&technology star of Zhujiang, Guangzhou city (2011J2200084); the project on the Integration of Industry and Research of Huizhou city (2011C010002004).

\section{References}

[1] L H. Keel and S P. Bhattacharyya, "Robust, fragile, or optimal[J]", Automatic Control, IEEE Transactions on, vol. 42, no. 8, (1997), pp. 1098-1105.

[2] J. Doyle, A. Packard and K. Zhou, "Review of LFTs, LMIs, and $\mu[C]$ ", Decision and Control, Proceedings of the 30th IEEE Conference on. IEEE, (1991), pp. 1227-1232.

[3] D. W. Gu, "Robust Control Design with MATLABo[M]", Springer, (2005).

[4] D. McFarlane and K. Glover, "A loop-shaping design procedure using Ho synthesis[J]", Automatic Control, IEEE Transactions on, vol. 37, no. 6, (1992), pp. 759-769.

[5] G. Zames and A K. El-Sakkary, "Unstable systems and feedback: The gap metric[C]", Proc. Allerton Conf., (1980), pp. 380-385.

[6] A. El-Sakkary, "The gap metric: Robustness of stabilization of feedback systems [J]", Automatic Control, IEEE Transactions on, vol. 30, no. 3, (1985), pp. 240-247.

[7] M. Vidyasagar, "Control System Synthesis: A Factorization Approach", MIT Press, Cambridge, (1985).

[8] G. Vinnicombe, "Frequency domain uncertainty and the graph topology[J]", IEEE Transactions on Automatic Control, vol. 38, no. 9, (1993), pp. 1371-1383.

[9] T T. Georgiou and M C. Smith, "Optimal robustness in the gap metric[J]", Automatic Control, IEEE Transactions on, vol. 35, no. 6, (1990), pp. 673-686.

[10] L. Qui and E. J. Davison, "Pointwise gap metrics on transfer matrices[J]", Automatic Control, IEEE Transactions on, vol. 37, no. 6, (1992), pp. 741-758.

[11] B. Liu, C. H. Wang and W. Li, "Gap metric and robust controller design in tracking systems[J]", Control and Decision, vol. 25, no. 11, (2010), pp. 1713-1718.

[12] K. M. Zhou and J. C. Doyle, "Essentials of robust control[M]", New Jersey: Prentice Hall, (1998).

[13] Y. Ochi and H. Kondo, "PID controller design based on optimal servo and v-gap metric[C]",American Control Conference (ACC), 2010. IEEE, (2010), pp. 1091-1096.

[14] J. Du and T. A. Johansen, "A gap metric based weighting method for multimodel predictive control of MIMO nonlinear systems[J]", Journal of Process Control, vol. 24, no. 9, (2014), pp. 1346-1357.

[15] D. Yubo, Y. Sai and L. Bin, "Structural Robustness and Multi-Model Control in Gap Metric[J]", International Journal of Control and Automation, vol. 6, no. 6, (2013), pp. 381-392. 
[16] J. Kennedy, "Particle swarm optimization[M]", Encyclopedia of Machine Learning. Springer US, (2010), pp. 760-766.

[17] T. Ganesan, P. Vasant and I. Elamvazuthy, "A hybrid PSO approach for solving non-convex optimization problems [J]", Archives of Control Sciences, vol. 22, no. 1, (2012), pp. 87-105.

[18] A U. Genç, "A state-space algorithm for designing Ho loop shaping PID controllers[J]”, Tech. Repot of Cambridge Univ., (2000).

[19] D.C. McFarlane and K. Glover, "Robust Controller Design using Normalised Coprime Factor Plant Descriptions", Springer Verlag, Lecture Notes in Control and Information Sciences, vol. 138, (1989).

[20] D.C. McFarlane and K. Glover, "A Loop Shaping Design Procedure using Synthesis", IEEE Transactions on Automatic Control, vol. 37, no. 6, June (1992), pp. 759- 769.

[21] R. Toscano, "Structured Controllers for Uncertain Systems: A Stochastic Optimization Approach[M]", Pringer, (2013).

[22] L. Qiu and K. Zhou, "Introduction to feedback control[M]", Upper Saddle River: Pearson Prentice Hall, (2009).

[23] L. Xianli and L. Bin, "Adaptive optimal robust control in gap metric[C]", Control and Decision Conference (CCDC), 2011 Chinese. IEEE, (2011), pp. 185-190.

[24] D. Bratton and J. Kennedy, "Defining a standard for particle swarm optimization[C]",Swarm Intelligence Symposium, 2007. SIS 2007. IEEE. IEEE, (2007), pp. 120-127.

[25] S. Skogestad and I. Postlethwaite, "Multivariable feedback control: analysis and design [M]", New York: Wiley, (2007).

[26] D. C. McFarlane and K. Glover, "Robust controller design using normalized coprime factor plant descriptions[J]", (1990).
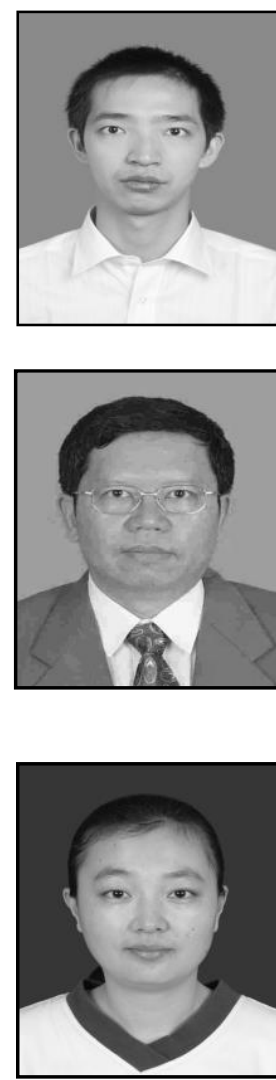

\section{Authors}

Xinghong QIAO. He was born in 1979. He received his M.S. degree in University of Chinese Academy of Sciences in 2008. He is a Ph.D. candidate in South China University of Technology. His research interests include robust optimization with applications in process systems engineering.

Email: qiaoxinghong@126.com

Fei LUO, He received his M.S and Ph.D. degrees in 1986 and 1993 from University of Science \& Technology Beijing, China. He is currently a professor with College of Automation Science and engineering, South China University of Technology, China. His research interests include His current research interests include nonlinear dynamical systems and control, nonlinear observer design, robust control, and applications.

Yuge XU. She received her M.S and Ph.D. degrees in 2003 and 2006 from South China University of Technology, China. She is currently a associate professor with College of Automation Science and engineering, South China University of Technology, China. Her research interests include robust control, controller design under risk specifications, robust and chance constrained optimization. 